\title{
Somatic Cell Nuclear Transfer in Oregon: Expanding the Pluripotent Space and Informing Research Ethics
}

\author{
Geoffrey P. Lomax* and Natalie D. DeWitt
}

\section{ABSTRACT}

In May, Oregon Health and Science University (OHSU) announced the successful derivation, by the Mitalipov laboratory, of embryonic stem cells by somatic cell nuclear transfer. This experiment was recognized as a "formidable technical feat" and potentially a key step toward developing cellbased therapies. The OHSU report is also an example of how a scientific breakthrough can inform research ethics. This article suggests ways that nuclear transfer embryonic stem cell lines may contribute to research ethics by adding rigor to studies addressing pressing research questions important to the development of cell-based therapies.

\section{Somatic cell nuclear}

\section{transfer and variability in the pluripotent space}

Sir William Osler described medicine as "a science of uncertainty and an art of probability" [101]. Uncertainty is a fundamental challenge in the development of cell-based therapies from pluripotential cells-human embryonic stem cells (hESCs), induced pluripotent stem cells (iPSCs), and nuclear transfer embryonic stem cells (NT-ESCs) [1]. Current clinical models envisage starting with a pluritpotent cell culture followed by considerable propagation and then differentiation to a therapeutic cell type or product [2]. Clin-

California Institute of Regenerative Medicine, San Francisco, California.

*(Correspondence: glomax@cirm.ca.gov) ical application of this model requires the development of standardized tests for product consistency, potency, stability, tumorigenicity, toxicity, and immunogenicity. However, standardization has proven challenging because cellular products are often heterogeneous and every type of stem cell can vary in its underlying biology and behavior [3].

A pressing question is the extent to which variability will affect the usefulness of cell products in regenerative medicine [4]. Variability exists both among and between cell lines and types being contemplated for clinical applications. Comparative studies among the same types of lines-for instance, hESC to hESC - and between types - $\mathrm{hESC}$ to iPSC - are ongoing. The categories, magnitude, mechanistic origins, and implications of these differences are the subject of intensive research and scholarly review [4-6].
Until recently, the human pluripotent space has been defined by hESCs and iPSCs (Fig. 1). While hESCs are often described as the gold standard for evaluating pluripotency and differentiation potential [102], iPSCs are viewed as having great promise, especially for autologous (derived or transferred from the same individual's body) transplantation to reduce the potential of histoincompatibility and risks from immune rejection [7]. Differences in differentiation potential and immunogenicity have been identified as critical factors for clinical applications and are being evaluated in comparative (hESC vs. iPSC) studies $[4,8]$.

One unique consideration for iPS cells is the extent to which the induction (reprogramming) process converts the somatic (adult) cell to an embryonic state. Experiments demonstrate that genes that are 
FIG. 1. The pluripotent space before nuclear transfer lines derivation.

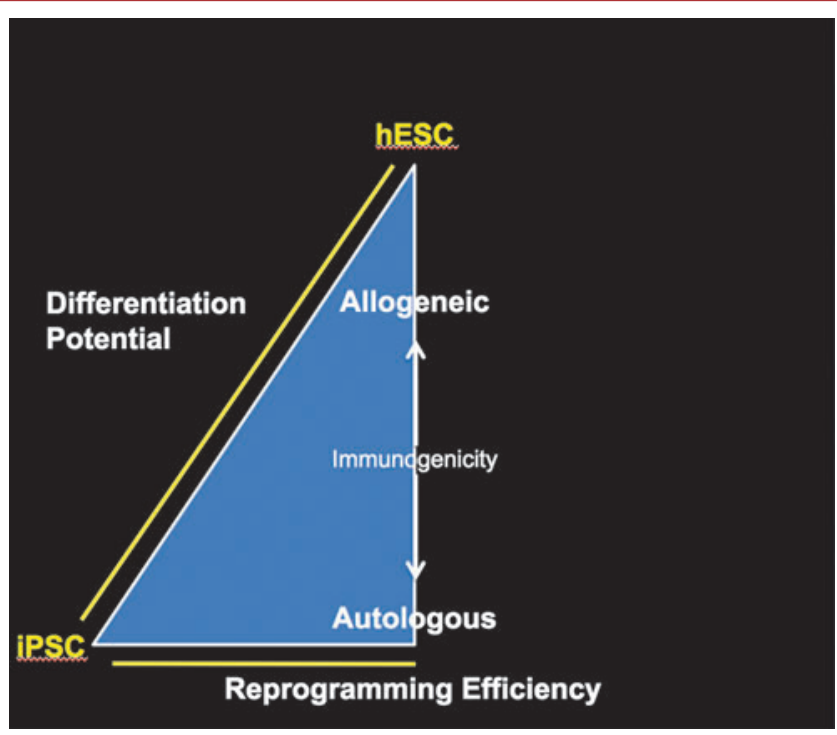

Prior to the derivation of NT-ESCs the pluripotent space included iPSCs and hESCs, area in blue. Comparative studies have focused on differentiation potential among and between cell types.

FIG. 2. The pluripotent space with nuclear transfer lines derivation.

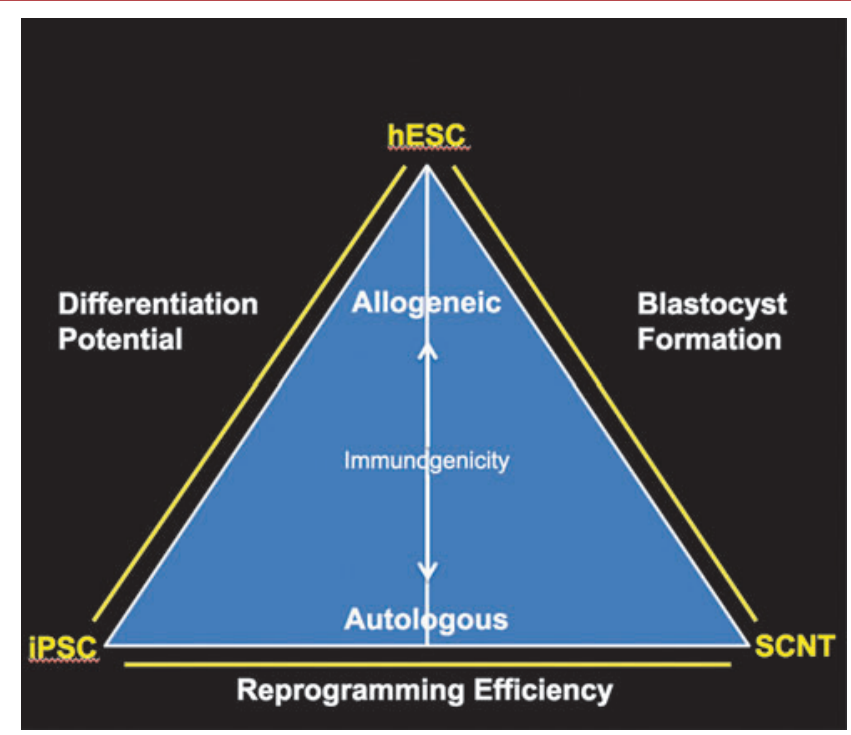

The derivation of NT-ESCs expands the pluripotent space, area in blue. Comparative studies can now expand their focus to include reprogramming efficiency and blastocysts characteristics among and between cell types.

normally silenced in hES cells can remain active in iPS cells [9]. The significance of this phenomenon, also known as incomplete DNA methylation, is not known, but it may impact subsequent propagation and differentiation.
The derivation of human NT-ESC lines now expands the pluripotent space (Fig. 2).* As Mitalipov suggests, NT-ESCs

${ }^{*}$ These figures are for illustrative purposes. Recent studies suggest rare somatic cells offer a new platform for the development of cell-based therapies [1]. However, the resulting NT-ESC lines also provide a comparator (iPSC vs. NT-ESC) that may be particularly useful for identifying factors relating to somatic cell reprogramming efficiency and effectiveness. Further, the Oregon Health and Science University (OHSU) team derived iPSCs from the NTESC somatic cell donor, so an iPSC-NTESC comparator exists for somatic cells obtained from the same individual, thus adding another important dimension to research aimed at optimizing the reprogramming process.

\section{Somatic cell nuclear transfer, cell-based therapies, and ethics}

The advantages of any particular cell type for clinical applications must be considered against other issues, including the nature of the disease and the timing of treatment. There are compelling reasons to advance a variety of therapeutic approaches involving multiple cell types, as evidenced by the numerous translational research studies supported by the California Institute for Regenerative Medicine's therapy development projects [103].

As therapy development progresses toward clinical trials, research ethics demand that risks to patients be minimized. Expanding the pluripotent space enables studies that were previously not possible, thus allowing consideration of clinical trial design based on more complete information. Susan Solomon, CEO of the New York Stem Cell Foundation, recently noted, "We currently conduct comparative studies between iPSCs and embryonic stem cells derived via nuclear transfer to understand similarities and differencesa crucial step before any cell therapies can reach patients" [104]. Comparative studies do not eliminate uncertainty, but they pro-

exhibiting pluripotent characteristics exist. See www.pnas.org/content/110/12/4598.full? sid=2b11e7c0-ef54-40dd-913e-283b53cb1edf 
vide additional knowledge. Issues and questions under consideration with regard to the therapeutic application of iPSCs are identified in Table 1.

To be ethical, clinical research must be conducted in a methodologically rigorous manner and have a favorable benefit-torisk ratio for the individual receiving treatment. Determining favorable benefit is particularly challenging with therapies originating from pluripotent cells because clinical experience is limited. Under these conditions and in the interest of methodological rigor, we are compelled to develop a body of evidence in preclinical studies performed in animal models. Testament to this point is the 22,000-plus-page Investigational New Drug application produced by Geron Corporation for its hESC-based GRNOPC1 product [10].

In the case of iPSC-based products, it is plausible that NT-ESC lines will play a substantial role in evaluating the efficacy of somatic cell reprogramming and differentiation, thus supporting benefit-torisk assessments. To date we have no clinical experience with iPSC-based products (one trial has been proposed [105]), suggesting, as with Geron's GRNOPC1 product, the need for extensive preclinical studies. One might argue that these conditions create an ethical imperative to incorporate knowledge gained from research involving NT-ESC lines into preclinical evaluation of iPSC-based products.

In a recent perspective on the state of stem cell science, Zachary Russ writes, "The roadblocks to research are quick to appear and slow to dissipate, but they are inevitably overcome" [106]. The example of somatic cell nuclear transfer for generating human cell lines is a case in point. After a decade of effort, OHSU researchers have reported an efficient and effective means of generating NT-ESC lines. As a result, the pluripotent space now includes a set of comparator lines for addressing a range of issues and questions related to safe and effective application

Table 1. Issues and Questions for Evaluating Therapeutic Applications

Reprogramming efficiency and effectiveness

- How does reprogramming efficiency vary between iPSC and NT-ESC, particularly for cells obtained from the same individual?

- Is epigenetic memory retained?

- Can differences be attributed to molecular factors?

- Can the efficiency and speed of iPSC reprogramming be improved?

Differentiation potential

- Does gene expression vary between iPSC and NT-ESC derived from the same individual?

- Does the ability to differentiate to mature cell types vary by source of line (e.g., hESC, iPSC, and NT-ESC)?

- What is the nature of this variation?

Genetic stability in culture

- How does the stability of NT-ESC compare to iPSC lines that have been reported to be genetically unstable in culture?

Engraftment

- Do NT-derived cell lines differ from hESC or iPSC lines in their detection by immune surveillance, which could impact the engraftment efficiency and durability of cells or derivatives?

hESC, human embryonic stem cell; iPSC, induced pluripotent stem cell; NT-ESC, nuclear transfer embryonic stem cell.

of cell-based therapies. This breakthrough comes just as cell-based therapies from pluripotent cells are entering clinical application. The additional knowledge provided by NT-ESC lines

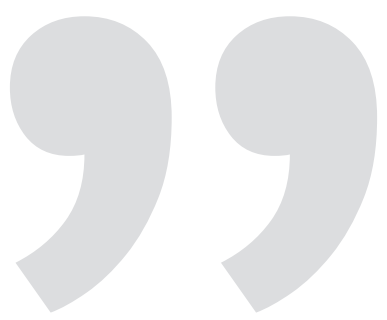

The pluripotent space now includes a set of comparator lines for addressing a range of issues related to safe and effective applications of cell based therapies. will support research ethics by enhancing the empirical basis for evaluating risks and benefits in future clinical trials.

\section{Author disclosure} statement

No competing financial interests exist.

\section{REFERENCES}

\section{Primary Literature}

1. Tachibana M et al. (2013). Human embryonic stem cells derived by somatic cell nuclear transfer. Cell 153:12281238.

2. Carpenter MK, J Frey-Vasconcells and MS Rao. (2009). Developing safe therapies from human pluripotent stem cells. Nat Biotechnol 27:606-613.

3. Strauss S. (2010). Geron trial resumes, but standards for stem cell trials remain elusive. Nat Biotechnol 28:989-990.

4. Cahan P and GQ Daley. (2013). Origins and implications of pluripotent stem cell 
variability and heterogeneity. Nat Rev Mol Cell Biol 14:357-368.

5. Cohen DE and D Melton. (2011). Turning straw into gold: directing cell fate for regenerative medicine. Nat Rev Genet 12:243-252.

6. Wu SM and K Hochedlinger. (2011). Harnessing the potential of induced pluripotent stem cells for regenerative medicine. Nat Cell Biol 13:497-505.

7. Chun YS, P Chaudhari and YY Jang. (2010). Applications of patient-specific induced pluripotent stem cells; focused on disease modeling, drug screening and therapeutic potentials for liver disease. Int J Biol Sci 6:796-805.

8. Pearl JI, LS Kean, MM Davis and JC Wu. (2012). Pluripotent stem cells: im- mune to the immune system? Sci Transl Med 4:164ps125.

9. Ohi Y et al. (2011). Incomplete DNA methylation underlies a transcriptional memory of somatic cells in human iPS cells. Nat Cell Biol 13:541-549.

10. Alper J. (2009). Geron gets green light for human trial of ES cell-derived product. Nat Biotechnol 27:213-214.

\section{Websites}

101. Wikiquote William Osler en.wikiquote .org/wiki/william_osler

102. Gawrylewski A. Embryonic stem cells still gold standard. The Scientist, June 13, 2008. www.the-scientist.com/? articles.view/articleno/26495/title/embry onic-stem-cells-still-gold-standard/
103. CIRM, Progress toward therapies. www.cirm.ca.gov/our-progress/progresstoward-therapies

104. Farrell J. Forbes, May 17, 2013. www .forbes.com/sites/johnfarrell/2013/05/17/vi able-human-embryonic-stem-cells-cre ated-by-cloning/

105. Mellough C. First iPSC clinical trials to take place in Japan. Stem Cells Portal. www.stemcellsportal.com/newsand-press/latest-news/571-first-ipscclinical-trials-to-take-place-in-japan .html

106. Russ Z. The human roots of stem cells. Genetic Engineering and Biotechnology News. www.genengnews.com/ gen-articles/the-human-roots-of-stemcells/4428/ 